\title{
Teaching evidence based medicine literature searching skills to medical students during the clinical years - a protocol for a randomised controlled trial
}

Dragan $\| \mathrm{Ii}^{\mathrm{1}^{*}}$, Katrina Tepper ${ }^{2}$ and Marie Misso ${ }^{3}$

\begin{abstract}
Background: Two of the key steps in evidence based medicine (EBM) are being able to construct a clinical question and effectively search the literature to source relevant information. No evidence currently exists that informs whether such skills should be taught to medical students during their pre-clinical years, or delivered to include both the pre-clinical and clinical years of study. This is an important component of curriculum design as the level of clinical maturity of students can affect their perception of the importance and uptake of EBM principles in practice.

Methods/Design: A randomised controlled trial will be conducted to identify the effectiveness of delivering a formal workshop in EBM literature searching skills to third year medical students entering their clinical years of study. The primary outcome of EBM competency in literature searching skills will be evaluated using the Fresno tool.

Discussion: This trial will provide novel information on the effectiveness of delivering a formal education workshop in evidence based medicine literature searching skills during the clinical years of study. The result of this study will also identify the impact of teaching EBM literature searching skills to medical students during the clinical years of study.
\end{abstract}

\section{Background}

Competency in evidence based medicine (EBM) provides clinicians with the ability to identify, evaluate and integrate evidence into clinical decision making. Two of the five critical steps in achieving competency in EBM are to (i) construct an answerable question from the clinical environment, and (ii) effectively and efficiently search the medical literature to identify the best available evidence to answer the question [1].

Most studies performed to date have focused on evaluating participants' critical appraisal skills in EBM. A systematic review of postgraduate teaching in EBM conducted in 2004 identified improvement in knowledge, critical skills, attitudes and behaviour; however it did not formally evaluate EBM skills in literature searching

\footnotetext{
* Correspondence: dragan.ilic@monash.edu

'Department of Epidemiology \& Preventive Medicine, School of Public

Health \& Preventive Medicine, Monash University, Victoria, Australia

Full list of author information is available at the end of the article
}

[2]. A systematic review of EBM teaching from the undergraduate and post-graduate perspective identified one study that reported an increase in formulating clinical questions and searching the literature skills in medical residents attending a formal EBM workshop [3,4]. Similarly, a trial in undergraduate medical students comparing a workshop to didactic EBM teaching reported an increase in skills to construct a clinical question and perform a literature search in students attending the workshop compared to the didactic teaching $[4,5]$.

Various training modules and courses in EBM have now commonly been implemented across medical schools worldwide [6]. The aim of such EBM programs is to provide an integration of knowledge, cognitive skills and behaviour that promotes life-long learning for future medical graduates [4]. Several studies have reported that training undergraduate medical students in EBM literature searching skills using a variety of 
interventions ranging from individual workshops to ongoing didactic lectures, is associated with an improvement in literature searching skills [5,7-9].

Whilst various studies have identified the positive impact of EBM workshops on students, none have identified whether specialised searching skills are more effectively taught to medical students solely during their preclinical or clinical years; or a combination of both. The level of clinical maturity of students can affect their perception of the importance and uptake of EBM principles in practice. A study of junior doctors' knowledge and beliefs in EBM identified a belief that EBM was an essential skill relevant to their clinical practice, despite few having partaken in formal training in the principles of EBM [10]. Conversely, first year medical students who have not been exposed to the clinical environment have reported to perceive EBM as a static discipline, which is not as relevant to clinical medicine [11].

It is important to identify when certain aspects of EBM, be it constructing a question, searching the literature or critically appraising evidence, should be delivered within the undergraduate program in order to ensure that students obtain a satisfactory level of competence in EBM and are able to implement it throughout their clinical years.

\section{Methods/Design}

Aim

The aim of this randomised controlled trial (RCT) is to determine the effectiveness of delivering a formal education workshop in evidence based medicine literature searching skills to undergraduate medical students during the clinical years of study. The specific objectives of the study are;

1. Compare the competency in EBM literature searching skills of medical students who participate in a formal workshop at the beginning of their clinical year, to medical students who have participated in introductory EBM literature search skills sessions in the preclinical years only; and

2. Determine students self perceived competency in EBM literature searching skills.

\section{Design}

This study is a randomised controlled trial conducted at Monash University, Australia.

\section{Settings and participants}

The study will be performed at the medical library at the Clayton campus of Monash University. Participants for the study will be third year medical students undertaking the Monash University MBBS degree at the Monash Medical Centre, Dandenong or Casey hospitals. Each of these hospitals are teaching hospitals, which form the 'Southern Health' network of hospitals.

The Monash University MBBS degree is a five year undergraduate course. Students within this course spend the first two years based at the University outside of the clinical environment. In the first two years of the MBBS course the students undertake introductory sessions in the principles of evidence based medicine literature searching. At the commencement of the third year students are solely based in the clinical environment, spending their entire teaching year based at one teaching hospital. From years three to five in the course students are only placed within a clinical learning environment.

The Monash MBBS undergraduate degree, and by extension the students, are comparable to similar MBBS degrees within Australia, and internationally, that train undergraduate students in the principles of EBM across preclinical and clinical years of the degree [12].

\section{Recruitment}

Third year medical students will be randomised to either a formal workshop in EBM literature searching skills as part of the EBM unit within the MBBS course or to a control group. Participants must be a third year Monash MBBS student to be eligible for the trial. Students who are unwilling to participate in the study will be excluded from the recruitment process.

\section{Randomisation}

Participants will be randomly assigned independently by the 'Southern Health' clinical site administrator by block randomisation to either the intervention or comparison groups. A computer random number generator will be used to generate a randomisation list in blocks of four (Figure 1).

\section{Intervention}

Students randomised to the EBM literature searching skills (EBM-LSS) workshop will receive a two hour workshop within the computer laboratory at the Hargrave-Andrew Library at the Clayton campus of Monash University. The EBM-LSS workshop will consist of a 30-minute formal presentation by the subject librarian, an interactive computer based searching session, and self-directed learning tasks (with the subject librarian to provide support when requested). Students randomised to the control group will not receive the workshop. Students in the control group will attend the EBM-LSS workshop once assessment of all outcomes across the intervention and control groups has been completed. 


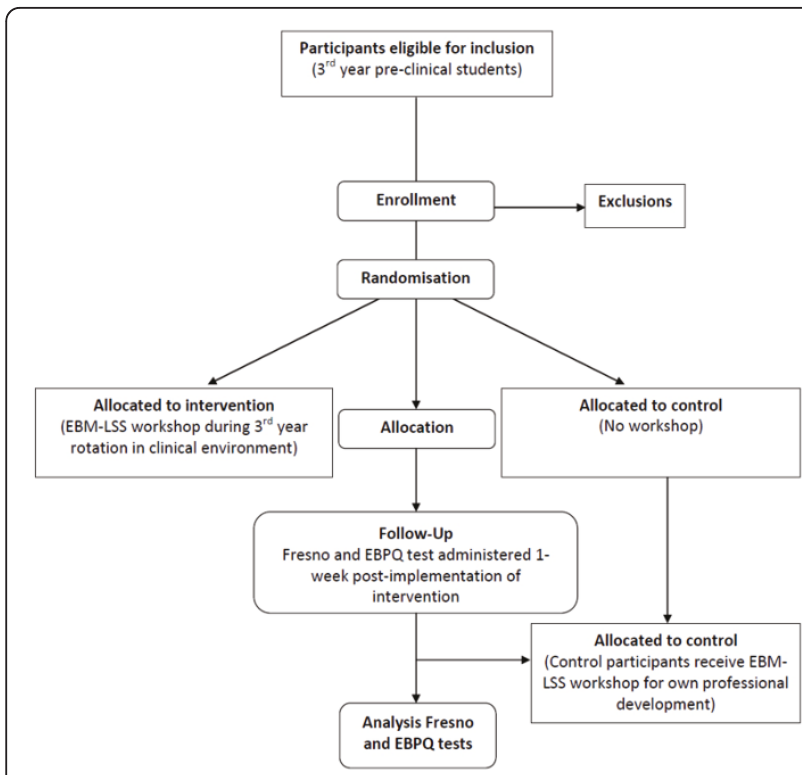

Figure 1 Flow diagram of proposed randomised controlled trial.

\section{Outcome measures}

The primary outcome to be measured in this study will be competency in EBM literature searching skills. This will be measured by using the previously validated Fresno tool [13]. Additionally, student self perceived competency in EBM literature searching skills will be assessed using the previously validated Clinical Effectiveness and Evidence Based Practice Questionnaire (EBPQ) [14]. These outcomes will be measured across both intervention and control groups 1-week post-implementation of the intervention.

\section{Blinding}

Blinding of investigators and participants is not possible as the subject librarian and students will be aware of the allocated arm. The outcome assessor and data analyst will be kept blinded to the allocation.

\section{Analyses}

\section{Sample size calculation}

We referred to a previous study, which implemented the Fresno test to assess searching skills, to estimate our sample size [15]. This previous study identified that a mean difference of 13 points on the Fresno test to be statistically significant in identifying competency between groups in effective EBM literature searching. A mean difference of 13 points, with a standard deviation of 10, is meaningful to discriminate between 'novice' and 'expert' users of EBM principles [13]. We determined that with a power of $90 \%$, alpha of 0.05 , the required sample size per each group is 21 , for a total sample size of 42 participants.
Multiple regression analysis will be used to determine the ability of certain variables (such as hospital site, previous exposure to EBM, perceived research ability and country of origin) to predict competency in EBM literature searching. We determine that with a power of $80 \%$, alpha set at 0.05 , and a medium effect size $\left(f^{2}=0.15\right)$, a sample size of 84 is required to detect a significant model. Therefore, accounting for a potential 10\% dropout rate, a minimum of 100 participants, 50 per each group, will be recruited for the study.

\section{Analyses}

Data will be analysed using the principle of intention-totreat. Mean difference in EBM literature searching skills competency between the intervention and control groups, as determined by the Fresno tool, will be explored using a Student's t-test. Additional analyses will include descriptive statistics to characterise participants in terms of self perceived competency in EBM literature searching skills. Linear and logistic regression will be used to analyse continuous and dichotomous data respectively.

\section{Ethical approval}

Ethical approval for this study was granted by the Monash University Human Research Ethics Committee in November 2010.

\section{Discussion}

The primary purpose of this RCT is to identify the effectiveness of delivering a formal workshop in EBM literature searching skills to third year medical students entering their clinical years of study. This study will also assess the impact of clinical maturity of medical students with respect to their knowledge and uptake of EBM literature searching skills. The trial will also provide valuable information with regard to the students' self perceived competency in EBM literature searching skills and what impact this has upon their actual competency.

Previous studies have suggested that clinical maturity, perceived relevance in the clinical environment and continued practice within this context may influence a student's competence in EBM skills $[10,11,16,17]$. This study has the potential to further explore these issues and provide novel information with regard to student competency in EBM skills, which may assist in the formulation and refinement of existing medical curricula within the EBM context.

\section{Acknowledgements}

We would like to thank Professor Upton and Dr Upton for the use of the EBPQ.

\section{Author details}

'Department of Epidemiology \& Preventive Medicine, School of Public Health \& Preventive Medicine, Monash University, Victoria, Australia. 
${ }^{2}$ Hargrave-Andrew Library, Monash University, Victoria, Australia. ${ }^{3}$ The Jean Hailes Foundation for Women's Health Research Unit, School of Public Health \& Preventive Medicine, Monash University, Victoria, Australia.

\section{Authors' contributions}

All authors have had substantial intellectual contribution to this protocol. DI and $\mathrm{KT}$ conceived the design of the study. DI wrote the first and final drafts of the protocol. KT and MM contributed to the drafting process of the protocol. All authors have contributed to revising the protocol for intellectual content. All authors have read and approved the final manuscript and given final approval for the manuscript to be published.

\section{Competing interests}

Dragan llic is the coordinator for the EBM unit within the Monash University MBBS program. The authors declare no other competing interests.

Received: 25 January 2011 Accepted: 28 July 2011

Published: 28 July 2011

\section{References}

1. Straus S, Glasziou P, Richardson W, Haynes B: Evidence-based medicine: how to practice and teach it Edinburgh: Churchill Livingstone: Elsevier; 2011.

2. Coomarasamy $\mathrm{A}$, Khan $\mathrm{K}$ : What is the evidence that postgraduate teaching in evidence based medicine changes anything? A systematic review. BMJ 2004, 329:1017.

3. Smith C, Ganschow P, Reilly B, Evans A, McNutt R, Osei A, Saquib M, Surabhi S, Yadav S: Teaching residents evidence-based medicine skills: a controlled trial of effectiveness and assessment of durability. Journal of General Internal Medicine 2000, 15:710-715.

4. Ilic D: Teaching evidence based practice: Perspectives from the undergraduate and post-graduate viewpoint. Annals Academy of Medicine Singapore 2009, 38:559-563.

5. Ghali W, Saitz R, Eskew A, Gupta M, Quan H, Hershman W: Successful teaching in evidence-based medicine. Medical Education 2000, 34:18-22.

6. Dinkevich E, Marksinson A, Ahsan S, Lawrence B: Effect of a brief intervention on evidence-based medicine skills of pediatric residents. BMC Medical Education 2006, 6:1.

7. Gruppen L, Rana G, Arndt T: A controlled comparison study of the efficacy of training medical students in evidence-based medicine literature searching skills. Academic Medicine 2005, 80:940-944.

8. Vogel $E$, Block $K$, Wallingford $K$ : Finding the evidence: teaching medical residents to search Medline. Journal of the Medical Library Association 2002, 90:327-330.

9. Rosenberg W, Deeks J, Lusher A, Snowball R, Dooley G, Sackett D: Improving searching skills and evidence retrieval. Journal of the Royal College of Physicians 1998, 32:557-563.

10. Hadley J, Wall D, Khan K: Learning needs analysis to guide teaching evidence-based medicine: knowledge and beliefs amongst trainees from various specialities. BMC Medical Education 2007, 7:11.

11. Astin J, Jenkins T, Moore L: Medical students' perspective on the teaching of medical statistics in the undergraduate medical curriculum. Statistics in Medicine 2002, 21:1003-1006.

12. Finkel $M$, Brown $H$, Gerber $L$, Supino P: Teaching evidence-based medicine to medical students. Medical Teacher 2003, 25:202-209.

13. Ramos K, Schafer S, Tracz S: Validation of the Fresno test of competence in evidence based medicine. BMJ 2003, 326:319.

14. Upton D, Upton P: Development of an evidence-based practice questionnaire for nurses. Journal of Advanced Nursing 2006, 53:454-458.

15. Kim S, Willett L, Murphy D, O'Rourke K, Sharma R, Shea J: Impact of an evidence-based medicine curriculum on resident use of electronic resources: A randomized controlled study. Journal of General Internal Medicine 2008, 23:1804-1808.

16. Bradley P, Oterholt C, Nordheim L, Bjorndal A: Medical students' and tutors' experiences of directed and self-directed learning programs in Evidence-Based Medicine: A qualitative evaluation accompanying a randomized controlled trial. Evaluation Review 2005, 29:149-177.

17. Dorsch J, Aiyer M, Meyer L: Impact of an evidence-based medicine curriculum on medical students' attitudes and skills. Journal of the Medical Library Association 2004, 92:397-406.

\section{Pre-publication history}

The pre-publication history for this paper can be accessed here: http://www.biomedcentral.com/1472-6920/11/49/prepub

doi:10.1186/1472-6920-11-49

Cite this article as: llic et al:: Teaching evidence based medicine literature searching skills to medical students during the clinical years a protocol for a randomised controlled trial. BMC Medical Education 2011 11:49.

\section{Submit your next manuscript to BioMed Central and take full advantage of:}

- Convenient online submission

- Thorough peer review

- No space constraints or color figure charges

- Immediate publication on acceptance

- Inclusion in PubMed, CAS, Scopus and Google Scholar

- Research which is freely available for redistribution 